\title{
LA RADIOGRAFÍA PANORÁMICA EN LA DETECCIÓN TEMPRANA DE ENFERMEDADES CARDIOVASCULARES ASINTOMÁTICAS: REVISIÓN DE LA LITERATURA
}

\author{
RUİZ-GARCÍA DE CHACÓN VILMA *
}

Recibido: $30 / 11 / 2015$
Aceptado: 05/12/2015

\section{RESUMEN}

Los accidentes cerebro vasculares (ACV) representan un grave problema de salud pública, ya que causan secuelas de importancia e inclusive la muerte. Aproximadamente el $60 \%$ de los sobrevivientes sufren discapacidad lo cual tiene altos costos involucrados en el tratamiento y la rehabilitación de los pacientes. Cerca del $20 \%$ de los ACV son el resultado de la separación o ruptura de una placa aterosclerótica en la región de la arteria Carótida. La identificación de placas ateromatosas en la región carotidea, a través de la radiografía Panorámica, representa un hallazgo de relevancia porque contribuye con el diagnóstico precoz de una lesión grave que puede causar daños irreversibles en la salud. Sin embargo, la evaluación correcta de esta radiografía aún representa un desafío para el odontólogo general. El propósito de este artículo es realizar una revisión de la literatura sobre la prevalencia de ateromas calcificados de arteria Carótida (ACAC) detectados en radiografías panorámicas de pacientes asintomáticos y en presencia de enfermedades sistémicas y periodontales, así como los factores de riesgo asociados. Del mismo modo revisar las estructuras radiopacas proyectadas en la zona de interés que podrían llevar a confusión en la identificación de los ACAC.

Palabras clave. Radiografía panorámica, placa aterosclerótica.

\section{ABSTRACT}

Cerebrovascular accidents (CVA) are a major public health problem, as they cause major consequences or death, Approximately $60 \%$ of survivors suffer disability wich has high costs involved in the treatment and rehabilitation of patients. About $20 \%$ of strokes are a result of separation or rupture of an atherosclerotic plaque in the carotid artery region. The identification of atheromatous plaques in the carotid region, through panoramic radiography, is a finding of relevance because it contributes to the early diagnosis of a serious injury that can cause irreversible damage to health. However, the correct assessment of this radiograph still represents a challenge to the general practitioner. The purpose of this article is to review the literature on the prevalence of calcified carotid artery atheroma (CCAA) detected on panoramic radiographs of asymptomatic patients and in the presence of systemic and periodontal diseases and associated risk factors. Similary check the radiopaque structures designed in the area of interest that could lead to confusion in the identification of the CCAA.

Keywords. Panoramic radiography, atherosclerotic plaque.

\section{INTRODUCCION}

Los accidentes cerebrovasculares (ACV) son la tercera causa de mortalidad en el mundo. Se calcula que para el 2050, en el primer mundo se incrementará el número de individuos afectados hasta en 59\%. En América Latina, el panorama epidemiológico es similar a los países industrializados por cambios en el estilo de vida; asimismo, es la tercera causa de años perdidos saludables.

EI ACV representa más de 150000 muertes por año en los Estados Unidos, solamente detrás de las enfermedades cardiovasculares y cáncer. Es un grave problema de salud pública, ya que causa la muerte y discapacidades físicas y / o mentales; aproximadamente el $60 \%$ de los sobrevivientes sufren discapacidad lo cual tiene altos costos involucrados en el tratamiento y la rehabilitación de los pacientes ${ }^{2}$. Aproximadamente el $20 \%$ de los ACV son el resultado de la separación o ruptura de una placa aterosclerótica en la región de la arteria Carótida ${ }^{3}$.

La identificación de placas ateromatosas en la región carotidea, a través de la radiografía panorámica, representa un hallazgo de relevancia porque contribuye con el diagnóstico precoz de una lesión grave que puede causar daños irreversibles en la salud del paciente. Sin embargo, la evaluación correcta de la radiografía panorámica aún representa un desafío para el cirujano dentista.

El propósito de este artículo es realizar una revisión de la literatura sobre la prevalencia de ateromas calcificados de arteria Carótida (ACAC) detectados en radiografías Panorámicas de pacientes asintomáticos y

* Asociación Peruana de Radiología Bucal y

Máxilofacial. Lima, Perú. 
en presencia de enfermedades sistémicas y periodontales, así como los factores de riesgo asociados. Del mismo modo revisar las estructuras radiopacas proyectadas en la zona de interés que podrían llevar a confusión en la identificación de las mismas.

\section{ATEROESCLEROSIS}

Los ACAC son un proceso patológico que ocurre en el lumen de las paredes de la arteria Carótida Común, cerca de su bifurcación. Existen una serie de factores que pueden considerarse como predisponentes para esta enfermedad, los cuales de modo general pueden clasificarse en intrínsecos y extrínsecos. En ocasiones, porciones del ateroma pueden liberarse y actuar como émbolos que ocluyen arterias de pequeño calibre. Cuando este fenómeno se da en vasos intra cerebrales causa accidentes cerebro vasculares (ACV) ${ }^{4}$. En vista de esto, se deben hacer todos los esfuerzos disponibles para reducir la prevalencia de los ACVs, entre estos la detección temprana de ACAC.

La radiografía panorámica es un examen imaginológico de rutina en odontología. En ella se pueden evaluar patologías de los tejidos duros, así como un gran número de condiciones de los tejidos blandos del macizo facial, entre ellas las mineralizaciones de las estructuras blandas (ACAC entre otras) ${ }^{5}$. Sin embargo, en ausencia de sintomatología y con las limitaciones propias de la técnica; esta no puede considerarse como gold estándar para este propósito.

La ultrasonografía Doppler es una técnica de imagen barata, accesible, exacta y no invasiva para evaluar ateroesclerosis de arteria Carótida. Esta técnica provee información sobre la naturaleza, morfología y grosor de la placa ateromatosa, así como el grado de estenosis de la arteria ${ }^{6}$ por lo que es el gold estándar para estas patologías.

Es innegable que el sedentarismo, el estrés y el consumo de cierto tipo de alimentos ha degenerado en un aumento alarmante de enfermedades tales como la obesidad, diabetes mellitus, hipertensión arterial, entre otras; las cuales aunadas a factores predisponentes intrínsecos de cada individuo, puede favorecer el desarrollo de patologías cardiovasculares las cuales en un gran porcentaje son absolutamente asintomáticas. Una vez que se ha producido un evento de ACV, el individuo que sobrevive a este queda con sus facultades restringidas. La recuperación y posterior reinserción a la sociedad es lenta y costosa. Según la Encuesta Nacional de Hogares del año 2013, el 66.3\% de asegurados corresponde al MINSA, EsSalud y FFAA ${ }^{7}$. Los gastos de curación y rehabilitación de estos pacientes se cargan directamente en un gran porcentaje en el presupuesto estatal, cosa que podría empezar a cambiar frente a un enfoque de detección temprana.

\section{GENERALIDADES}

Vilar refiere que en 1981 Friedlander y Lande reportaron por primera vez la presencia de calcificaciones en la región de la arteria Carótida Común en radiografías Panorámicas realizadas como parte del diagnóstico odontológico de rutina, y por lo tanto, se puede identificar pacientes asintomáticos con riesgo de sufrir un evento cardiovascular adverso ${ }^{8}$.

Radiográficamente, los ACAC se presentan como masas radiopacas irregulares, circulares, vérticolineares o heterogéneas, unilaterales o bilaterales. Se proyectan usualmente póstero superior al ángulo de la mandíbula, aproximadamente en el margen inferior de la tercera vértebra cervical cerca al hueso hioides (Figuras 1 y 2$)^{9}$, $10,11,12$

\section{PREVALENCIA DE ACAC EN RADIOGRAFÍAS PANORÁMICAS Y FACTORES DE RIESGO ASOCIADOS}

A partir de los hallazgos de Friedlander, se han realizado múltiples investigaciones relacionadas a con este descubrimiento, inclusive en algunos países asiáticos se ha adoptado el reporte de ACAC en radiografías Panorámicas como una herramienta establecida de prevención en salud pública (Tabla Nº1).

Lee y su equipo ${ }^{13}$ investigaron la asociación de ACAC en Panorámicas y la determinación de ateroesclerosis con ultrasonografía. Hallaron una prevalencia del $6 \%$ a predominio del sexo femenino. Los factores asociados fueron la edad, la glucosa aumentada y la hiperlipidemia. Imanimoghaddam y cols. $^{6}$ realizaron una investigación con el fin de identificar pacientes con riesgo de ACV mediante la detección de ACAC en radiografías Panorámicas y evaluaron los factores de riesgo asociados. Encontraron una prevalencia de 1.97\% a predominio del sexo femenino.

De ellos, sólo 15 accedieron a realizarse la ecografía Doppler, hallando que 10 presentaban la patología de modo bilateral y 5 unilateral. En cuanto a los factores de riesgo, sólo la edad mostró una asociación significativa. Sens y cols. ${ }^{14}$ trabajaron evaluando la sensibilidad y exactitud de la Panorámica en la identificación de ACAC y compararon los resultados con exámenes de ecografía Doppler. Luego de examinar radiografías de pacientes 
mayores de 40 años por un periodo de 11 meses, llegaron a la conclusión de que 21 imágenes presentaban características innegables de ACAC.

De estas 17 fueron confirmadas por ecografía Doppler, siendo la prevalencia de $80.95 \%$, concluyendo que la radiografía Panorámica es sensible para la detección de estas patologías. Baryam y colaboradores ${ }^{15}$ trabajaron evaluando 4106 radiografías panorámicas en búsqueda de ACAC y correlacionaron sus hallazgos con la literatura. Encontraron una prevalencia de $2.14 \%$ a predominio del sexo femenino, Concluyeron que las radiografías Panorámicas pueden detectar ACAC. Alves y equipo ${ }^{16}$ realizaron una revisión de la literatura en la cual se concluyó que las ACAC van en un rango de $0.43 \%$ a $9.4 \%$ dependiendo de la población estudiada. Cuando existen enfermedades sistémicas esta prevalencia se eleva hasta $38.8 \%$.

Es más común en mujeres y es detectable en radiografías panorámicas. Baumann y colaboradores ${ }^{17}$ realizaron una investigación cuyo objetivo fue determinar si los ACAC pueden ser hallazgos en radiografía Panorámica y si esta sospecha puede ser confirmada mediante ecografía. La prevalencia fue de $3.59 \%$. Entre los factores de riesgo considerados, se encontró relación con la edad. Khambete y su equipo ${ }^{18}$ realizaron una investigación que pretendió determinar si la radiografía Panorámica es un método confiable para la detección de ACAC comparada con la ultrasonografía. Encontraron una prevalencia del 34\%. La sensibilidad y especificidad de la radiografía Panorámica digital fue del $76 \%$ y $98.66 \%$ respectivamente; llegando a la conclusión de que es una herramienta válida de detección de ACAC. Sisman y colaboradores ${ }^{19}$ desarrollaron un estudio que tuvo como objetivo determinar de modo retrospectivo la presencia de ACAC en radiografías Panorámicas, además de registrar factores de riesgo asociados. Encontraron una prevalencia del $5.06 \%$ a predominio del sexo femenino. Entre los factores de riesgo que estaban asociados se pueden citar la hiperlipidemia, la hipertensión arterial, la diabetes mellitus y el ser fumador.

Yoon y su equipo 20 trabajaron en una investigación cuyo propósito fue diagnosticar la exactitud de la radiografía panorámica en la detección de ACAC usando la tomografía computarizada como gold estándar. Encontraron una prevalencia de ACAC del $15 \%$ en Panorámica y del $40.9 \%$ en tomografía. Ezoddini y colaboradores $^{21}$ desarrollaron una investigación que tenía como objetivo evaluar la eficacia de la radiografía Panorámica digital en la detección de ateroesclerosis. Hallaron una prevalencia de $2.43 \%$ a predominio del sexo femenino. Entre los factores de riesgo evaluados se determinó que la hipertensión arterial tiene asociación con la presencia de ACAC.

\section{PREVALENCIA EN ENFERMEDADES SISTÉMICAS}

Ante la presencia de enfermedades sistémicas el porcentaje de prevalencia de los ACAC se eleva hasta casi un $40 \%$. Esto se debe a que al existir alteraciones en el equilibrio funcional de los diferentes sistemas y aparatos, la normal captación y liberación de los fosfatos de calcio se afecta de modo importante, favoreciendo los procesos de mineralización de los tejidos blandos en general, entre estos los ACAC; por lo que ante la existencia de enfermedades de fondo, la ateroesclerosis debe ser estudiada para una pronta identificación y tratamiento adecuado. (Tabla $\mathrm{N}^{\circ} 2$ ).

Hamedani y colaboradores ${ }^{22}$ investigaron la relación entre la osteoporosis, los ACAC y el proceso estilo hioideo elongado. Trabajaron con 78 mujeres en las que hallaron que el $70 \%$ con baja densidad de masa ósea presentaron al menos un proceso estilo hioideo elongado. La presencia de procesos estilo hioideos elongados de manera bilateral estaba asociada a la presencia de ACAC. Concluyeron que los individuos mayores con baja densidad de masa ósea y procesos estilo hioideos elongados tienen mayor riesgo de sufrir un evento cardiovascular adverso. Friedlander y colaboradores ${ }^{23}$ publicaron una investigación que tuvo como objetivo comparar la extensión de la calcificación de la aorta abdominal en radiografías laterales de la columna lumbar en pacientes con lesiones de ACAC presentes y ausentes en sus radiografías panorámicas. Las pacientes con ACAC visibles en radiografías panorámicas demostraron tener una asociación significativa con la calcificación de la aorta abdominal. El mismo autor con otro equipo de trabajo ${ }^{24}$ realizó un estudio para determinar la prevalencia y severidad de las calcificaciones del arco aórtico como hallazgo incidental en radiografías de tórax de mujeres post menopaúsicas que además tuvieron el hallazgo de ACAC en sus radiografías panorámicas. Además determinaron los factores de riesgo asociados. Hallaron 36 imágenes de ateromas (6.3\%), sin embargo no se encontró asociación significativa entre la presencia de ateromas y calcificaciones del arco aórtico a diferencia del estudio previo; pero se pudo determinar que esta última condición está ligada a la edad. Uthman y colaboradores ${ }^{25}$ publicaron una investigación que tuvo como objetivo evaluar la presencia de estenosis de la arteria Carótida con o sin calcificación en mujeres posmenopáusicas con hipertensión o diabetes. Hallaron una prevalencia de $1.7 \%$ en hipertensión y $1.3 \%$ en diabetes, con lo que concluyeron que estas patologías tienen una asociación significativa para la presencia de ACAC. David y cols. ${ }^{26}$ también trabajaron con mujeres posmenopaúsicas, a diferencia de Friedlander encontraron una prevalencia de ACAC del $43.2 \%$. De los factores de riesgo estudiados, sólo la hipertensión y la hiperlipidemia demostraron estar asociados de forma significativa.

Posteriormente Friedlander y cols. ${ }^{27}$ publicaron una investigación realizada en mujeres posmenopáusicas, el objetivo fue evaluar la relación entre ACAC en radiografías panorámicas y las imágenes de densidad ósea 
mineral de exámenes de densitometría ósea. Trabajaron con 20 mujeres de raza blanca y 24 de raza negra, llegando a la conclusión de que en presencia de ACAC la densidad ósea es menor. No hallaron asociación entre la presencia de ACAC y la raza.

Guerreiro y colaboradores ${ }^{28}$ publicaron los resultados de su investigación la cual tuvo como objetivo determinar la prevalencia de ACAC en radiografías Panorámicas de pacientes HIV positivos. Los investigadores hallaron una prevalencia del $8.33 \%$. Al correlacionar las variables de estudio, se halló que los pacientes que eran tratados con la combinación liponavir/ritonavir presentaron ateromas calcificados de arteria carótida en un 40.9\%. Concluyeron que los pacientes HIV positivos que reciben tratamiento con anti retrovirales incrementan el riesgo de enfermedades cardio vasculares debido a los desórdenes metabólicos originados por estos fármacos, lo que incrementa la prevalencia de factores sistémicos de riesgo.

Friedlander y cols. ${ }^{29}$ publicaron un estudio en el que investigaron la prevalencia de ACAC en pacientes con hiperparatiroidismo primario. Además identificaron factores de riesgo asociados. Hallaron ACAC en 24 pacientes (40\%). En cuanto a los factores de riesgo asociados, encontraron que la hipertensión y la dislipidemia

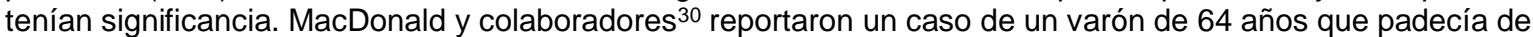
hiperparatiroidismo terciario como consecuencia de una enfermedad renal crónica, la cual le generaba hipercalcemia e hiperfosfatemia. Ellos coinciden con Friedlander en que las condiciones sistémicas de los pacientes producto de las condiciones derivadas de enfermedades concomitantes favorecen el desarrollo de ACAC. Gokce y colaboradores ${ }^{31}$ realizaron una investigación sobre la presencia de ACAC en radiografías Panorámicas de pacientes con enfermedad renal terminal tratados con diálisis peritoneal. Hallaron una prevalencia de $27.4 \%$ de ACAC.

Este estudio concuerda con los anteriores en que las condiciones sistémicas elevan de modo significativo el porcentaje de prevalencia de ACAC. Ahmad y colaboradores ${ }^{32}$ trabajaron con pacientes con tratamiento de hemodiálisis para determinar la relación de la máxima y media del grosor de la íntima de la carótida y los factores de riesgo en pacientes dializados. Concluyeron que en los pacientes dializados, los factores de riesgo cardiovascular son diferentes, en concordancia con los estudios anteriores.

Gunnarsson y cols. ${ }^{33}$ publicaron una investigación longitudinal que tuvo como objetivo determinar la asociación entre apnea obstructiva del sueño, grosor de la capa intima de la arteria Carótida y ACAC. Concluyeron que el apnea obstructiva del sueño se asocia al incremento del grosor de la capa íntima de la arteria y la presencia de ACAC. Por otra parte Chang y colaboradores ${ }^{34}$ propusieron la hipótesis de que los pacientes con síndrome $Z$ tienen mayor prevalencia de ateromas calcificados de Carótida que aquellos que sólo sufren de apnea obstructiva del sueño. Los investigadores hallaron que los pacientes con apnea obstructiva del sueño y síndrome metabólico concomitante (ambas condiciones juntas constituyen el síndrome Z) tuvieron mayor prevalencia de ateromas.

Pornprasertsuk y cols. ${ }^{35}$ realizaron una investigación cuyo objetivo fue detectar la sensibilidad y especificidad de la radiografía Panorámica en la detección de ACAC en pacientes con síndrome metabólico. Llegaron a la conclusión de que las radiografías panorámicas tienen sensibilidad moderada y alta especificidad. Por otra parte Tofangchiha y colaboradores ${ }^{36}$ investigaron la frecuencia de ACAC en pacientes con diabetes tipo II en individuos sanos en radiografías Panorámicas. Concluyeron que en pacientes con diabetes la frecuencia de ACAC es mayor, concordando en el estudio de Pornparsetuk en donde los factores metabólicos juegan un rol importante.

Khosropanah y cols. ${ }^{37}$ realizaron un estudio para determinar la utilidad de la radiografía Panorámica para detectar ACAC en pacientes con y sin enfermedad coronaria; así como el grado de concordancia entre la radiografía Panorámica y la ultrasonografía Doppler. Llegaron a la conclusión de que dada la baja sensibilidad y valor predictivo de la radiografía Panorámica, ésta no debe ser considerada como un método exacto para la detección de ACAC.

En el año 2009 Friedlander y cols. ${ }^{38}$ reportaron un caso de ACAC inducida post tratamiento de radiación por un carcinoma de células escamosas. Los investigadores proponen que la radioterapia acelera la enfermedad ateroesclerótica presente.

\section{ENFERMEDAD PERIODONTAL Y ENFERMEDAD CARDIOVASCULAR}

Desde hace ya varios años se ha evidenciado la relación existente entre la enfermedad periodontal y la cardiovascular. El proceso inflamatorio que acompaña a los problemas periodontales repercute directamente en el aumento del grosor de la capa íntima de los vasos sanguíneos, favoreciendo las condiciones para la aparición y desarrollo de ACAC. La evidencia científica ha demostrado que cuando se trata la enfermedad local 
de modo apropiado, la inflamación disminuye y el grosor de la capa íntima se reduce; revertiéndose las condiciones poco favorables que se presentaban anteriormente.

Ramesh y cols. ${ }^{39}$ reportaron una investigación en la cual se pretendió determinar la correlación entre la presencia de ACAC, el índice mandibular cortical y la pérdida ósea periodontal en radiografías Panorámicas. Evaluaron 185 panorámicas con ACAC y 234 sin ACAC, hallando una coincidencia significativa entre la presencia de ACAC y osteopenia, más no entre la presencia de ACAC y pérdida ósea periodontal. De otro lado, David y colaboradores ${ }^{40}$ estudiaron la relación entre los ACAC y pérdida ósea periodontal evaluada en panorámicas, hallando que la media de pérdida de altura ósea en ausencia de ACAC fue de $22.02 \mathrm{~mm}$, mientras que en presencia de ACAC fue de $28.30 \mathrm{~mm}$.

Shin y cols, publicaron una investigación ${ }^{41}$ en la cual quisieron asociar la enfermedad periodontal y el número de dientes perdidos con la ateroesclerosis sub clínica en población adulta coreana. Encontraron que el número de dientes perdidos está asociado con el engrosamiento de las capas media e íntima de la arteria Carótida Común, mientras que el sangrado al sondaje se asoció al engrosamiento de las capas media e íntima de la Carótida Común sólo en mujeres. El mismo año, Yu y colaboradores ${ }^{42}$ pretendieron probar la asociación entre la ateroesclerosis clínica /sub clínica y el estado periodontal. Se halló asociación con la hiperglicemia y la pobre higiene oral con el aumento de grosor de la íntima arterial y la presencia de placas ateromatosas. Morado y colaboradores ${ }^{43}$ realizaron una investigación de cohortes con el objetivo de relacionar el grado de ateroesclerosis de Carótida y la severidad de la periodontitis. Concluyeron que mientras más severo sea el caso de periodontitis más estará relacionado a la presencia de ateroesclerosis, coincidiendo con las investigaciones antes mencionadas. Puhar y colaboradores ${ }^{44}$ reportaron su investigación la cual tenía por propósito determinar la relación entre la condición periodontal y el grosor de la capa íntima de la arteria Carótida Común. Se tomaron en cuenta también los factores de riesgo asociados. Se halló que los cambios ateroescleróticos de la Carótida se pueden asociar a la periodontitis crónica. No encontraron asociación con los factores de riesgo a diferencia del estudio de $\mathrm{Yu}$. Previamente, Chin y colaboradores ${ }^{45}$ examinaron la relación entre edentulismo y la ateroesclerosis sub clínica en población coreana. Siendo la enfermedad periodontal crónica una condición inflamatoria común y constituyéndose como una de las principales causas de edentulismo; los autores postulan una relación directa entre esta y el grosor de la capa íntima de la Carótida y el consecuente desarrollo de ateroesclerosis. Sus hallazgos reportaron que más de la mitad de los individuos presentaban pérdida de dientes. Hábitos como beber y fumar no estuvieron asociados, coincidiendo con la investigación de Puhar y colaboradores. Hallaron relación entre la pérdida de dientes y el aumento del grosor de la capa íntima de la Carótida, concordando con el estudio de Shin. Los autores concluyen que ante este hallazgo, los pacientes con problemas periodontales crónicos padecen también de ateroesclerosis sub clínica.

En el año 2009 Cairo y cols. ${ }^{46}$ realizaron una investigación que tuvo como propósito evaluar el potencial de algunas variables periodontales en prevenir la ateroesclerosis subclínica y la inflamación sistémica en adultos jóvenes con periodontitis severa comparados con individuos sanos. Encontró que en pacientes periodontales la profundidad del sondaje periodontal fue predictor de la inflamación de la íntima de la Carótida y el sangrado al sondaje fue predictor de niveles elevados de proteína reactiva. El mismo equipo de trabajo publicó una investigación similar en el año $2008^{47}$ cuyo propósito fue evaluar la asociación entre periodontitis severa y ateroesclerosis sub clínica en pacientes menores de 40 años. Encontraron asociación entre ambas variables en pacientes jóvenes sistémicamente saludables.

Vaish y cols. ${ }^{48}$ publicaron un artículo de revisión cuyo objetivo fue discutir las hipótesis que explicarían la asociación entre periodontitis y enfermedades cardiovasculares. Consideraron factores como la relación entre la enfermedad periodontal y la enfermedad cardíaca, posibles mecanismos de asociación entre enfermedad periodontal y enfermedad cardiovascular, rol de las bacterias, incremento del fibrinógeno y elementos celulares blancos de la sangre, rol de los monocitos en enfermedad periodontal y ateroesclerosis, rol de la proteína C reactiva, rol de las proteínas de golpe de calor y factores de riesgo compartidos. Llegaron a la conclusión de que existe evidencia sustancial para relacionar la enfermedad periodontal y la enfermedad cardiovascular, y que los odontólogos deben reconocer la relevancia de la salud oral en la salud general y educar y motivar a sus pacientes. En otro artículo de revisión publicado el 2009 por Tonetti49, se expuso la importancia de los ensayos clínicos de intervención para establecer una relación de causalidad entre enfermedad periodontal y eventos cardiovasculares. Se revisó ensayos clínicos de intervención destinados a mejorar la inflamación sistémica, la disfunción endotelial, la ateroesclerosis carotidea y los eventos cardiovasculares. El autor concluye que la evidencia es consistente en que la periodontitis severa generalizada causa inflamación vascular sistémica y disfunción endotelial. Dados los efectos sistémicos de la periodontitis, su tratamiento y prevención pueden contribuir a la prevención de la ateroesclerosis. 


\section{DIFERENCIAS DE OTRAS RADIOPACIDADES ANATÓMICAS Y PATOLÓGICAS}

La deposición de sales de calcio normalmente ocurre en el cuerpo humano. Cuando esta ocurre de manera desorganizada en los tejidos blandos, se denomina calcificación heterotrópica. Estas mineralizaciones son comúnmente hallazgos radiográficos y es importante saber identificarlas de radiopacidades anatómicas y patológicas 50 para determinar la necesidad de tratamiento o de otros métodos de investigación. Se clasifican en tres categorías: distrófica: en el interior de tejido degenerado, idiopática: deposición de calcio en los tejidos normales, metastásica: en tejido normal a consecuencia del aumento de los niveles de calcio y fosfato ${ }^{51}$.

Vengalath y cols. ${ }^{52}$ desarrollaron una investigación cuyo objetivo fue determinar la prevalencia de calcificaciones visibles de tejidos blandos en la región orofacial en radiografías Panorámicas. Encontraron un total $8 \%$ de calcificaciones. Determinaron que las mujeres presentan mayor prevalencia de calcificaciones de tejidos blandos y que la ACAC fue la más prevalente en general. Bayer y colaboradores ${ }^{53}$ tuvieron como propósito mostrar el potencial de la radiografía panorámica para detectar hallazgos radiopacos en la región carotidea. A diferencia de Vengalath hallaron un porcentaje de $4.8 \%$ de hallazgos radiopacos compatibles con lesiones ateroescleróticas. La mayor parte de estas se presentaron en mujeres, al igual que en el estudio de Vengalath.

Saga y cols. ${ }^{54}$ publicaron una investigación que tuvo por objetivo determinar la localización de las calcificaciones de tejidos blandos similares a ACAC observadas en la región cervical de las radiografías panorámicas. Para esto prepararon 17 especímenes (cabeza y cuello de cadáveres) con esferas radiopacas posicionadas en estructuras anatómicas en los lugares correspondientes a las calcificaciones.

Se tomó una radiografía Panorámica a cada uno. Llegaron a la conclusión que el $75 \%$ de los examinadores indicaron correctamente la referencia de la bifurcación de la arteria Carótida, y el $79.2 \%$ señaló al cartílago Tritíceo como si fuese ACAC; los ACAC no son las únicas entidades que producen imágenes radiopacas en las área laterales de las radiografías panorámicas, por lo que es importante conocer estas estructuras para su correcta identificación. Ahmad y colaboradores ${ }^{55}$ desarrollaron una investigación para determinar la prevalencia del cartílago Tritíceo en las radiografías Panorámicas de personas mayores de 40 años, la distribución por sexo de quienes presentaban esta calcificación y cómo es que la forma de este cartílago calcificado difiere de los ACAC. Concluyeron que se presenta en un $5 \%$ de hombres y $12 \%$ de mujeres, tiene una forma oval con bordes lisos, mientras que los ACAC son mayormente lineares.

Garay ${ }^{56}$ publicó una revisión de la literatura de calcificaciones de tejidos blandos próximos al ángulo mandibular. Al igual que Saga, ante la gran variedad de radiopacidades proyectadas en el área de interés, recomiendan conocerlas de modo que su identificación sea más exacta. Alves y colaboradores ${ }^{57}$ reportaron un caso que presentó simultáneamente tonsilolitos y ACAC bilaterales. Se resaltó la importancia de observar cuidadosamente las radiografías Panorámicas para detectar calcificaciones distróficas del macizo facial, las cuales una vez identificadas serán de gran utilidad en el diagnóstico integral de la salud del paciente y su posterior derivación en caso de necesidad.

Manzi y colaboradores ${ }^{11}$ reportó un caso para el cual realizó una revisión de la literatura. En ella describió las calcificaciones patológicas y anatómicas que se proyectan en el área de interés y que podrían confundirse con ACAC. Entre las últimas están el cuerno mayor del hueso hioides, tejido blando de la lengua y el pabellón auricular, la epiglotis, los ligamentos estilo mandibulares y estilo hioideos. Los autores coinciden con las investigaciones antes mencionadas en donde se resalta la importancia de identificar estas estructuras anatómicas y patológicas para evitar confusiones y diagnósticos errados.

\section{CONCLUSIÓN}

La prevalencia de ACAC identificados en radiografías Panorámicas puede variar de $0,43 \%$ a 9.4\% dependiendo de la edad y estilos de vida de la población estudiada. En individuos con enfermedades sistémicas la prevalencia es más alta que en la población en general, alcanzando valores de alrededor de $38.8 \%$. Los factores de riesgo que han demostrado asociación con esta condición son en orden descendente la edad, la hipertensión arterial, la hiperlipidemia y la diabetes mellitus.

Las imágenes radiopacas compatibles con ACAC identificadas en radiografía Panorámica son más comunes en mujeres y pueden ocurrir de modo unilateral o bilateral, sin preferencia de lado. Sin embargo se debe tener en cuenta las mineralizaciones distróficas que podrían originar diagnósticos errados. 
Además, se puede concluir que el ACAC puede demostrarse en radiografías Panorámicas, siendo esta una herramienta muy importante para la detección temprana de los mismos. Para esto se debe entrenar a los odontólogos en la identificación de radiopacidades que sugieran la presencia de estas patologías vasculares. No obstante, siempre es necesario referir al paciente al servicio de Cardiología para confirmar los hallazgos, determinar la real extensión de la enfermedad y establecer el tratamiento correspondiente.

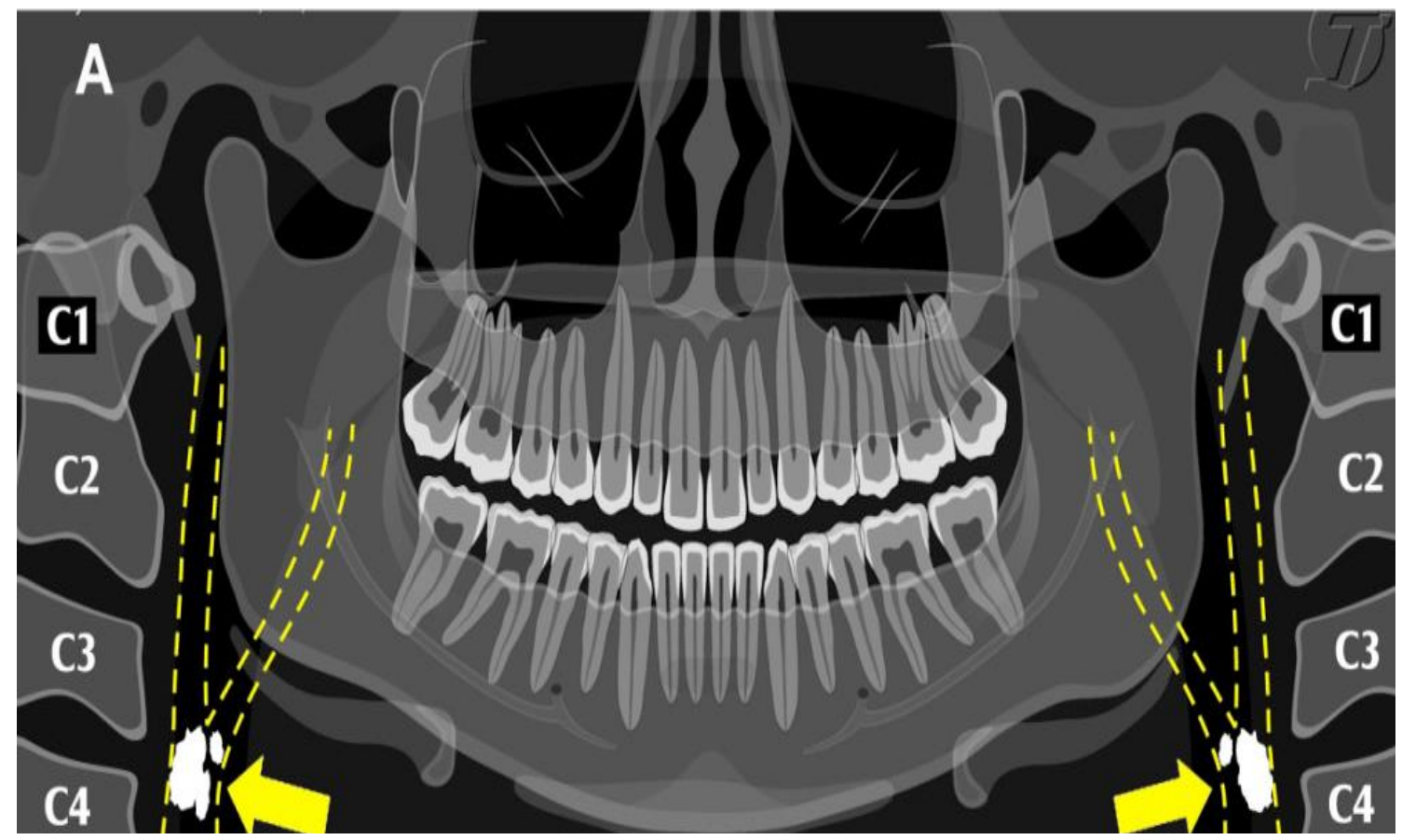

Fig.1. Localización de los ACAC en radiografía Panorámica.

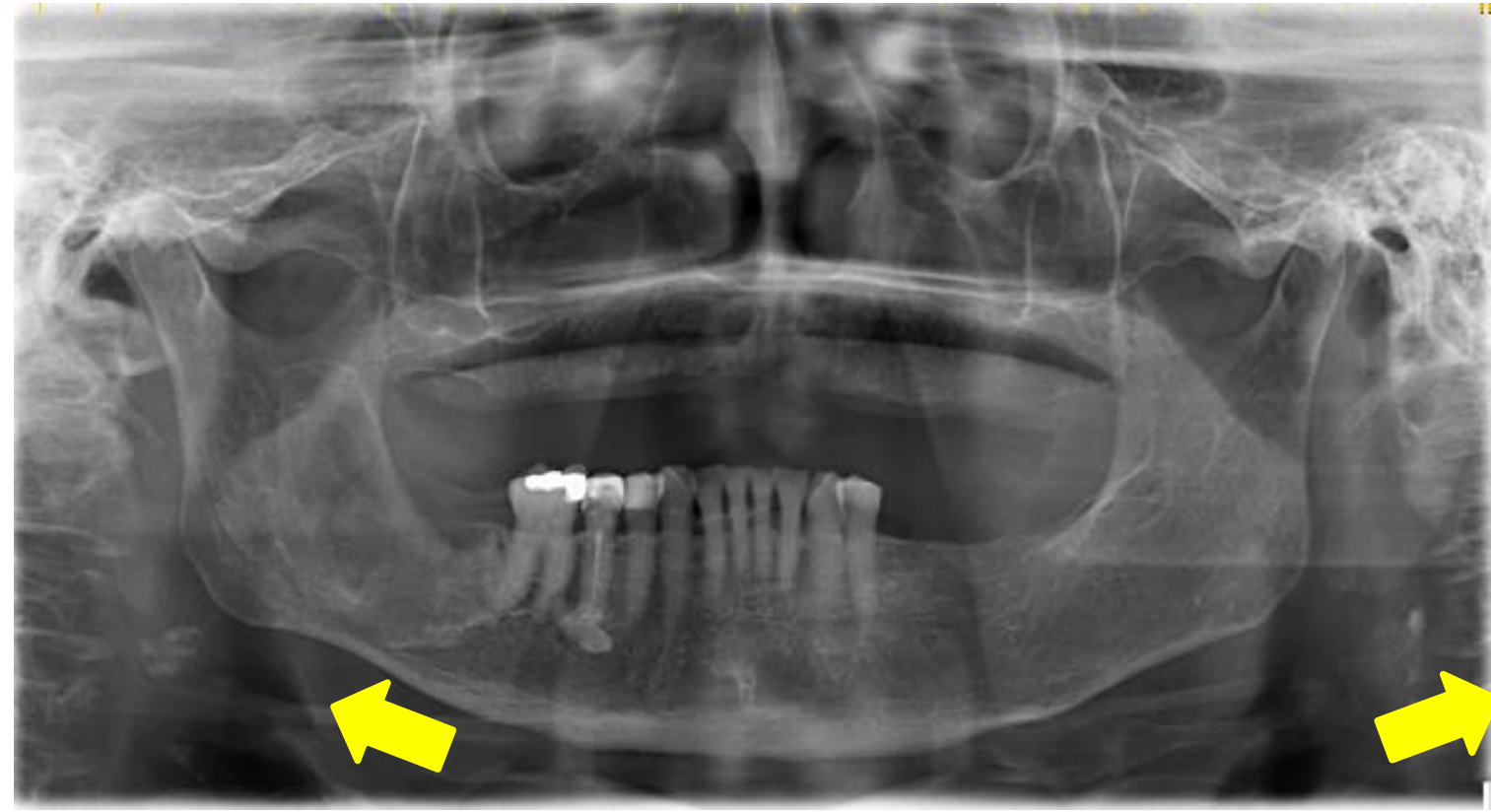

Fig. 2. Radiografía Panorámica mostrando ACAC. FUENTE: Propia 
Tabla 1. Prevalencia de ACAC en radiografías Panorámicas y factores de riesgo

\begin{tabular}{|c|c|c|c|c|c|c|c|c|c|c|c|c|c|c|}
\hline $\begin{array}{l}\frac{\tilde{u}}{0} \\
\stackrel{0}{3} \\
\stackrel{3}{\varangle}\end{array}$ & $\begin{array}{l}\stackrel{x}{凶} \\
\sim\end{array}$ & $\begin{array}{l}\bar{D} \\
\frac{\pi}{0} \\
\text { w }\end{array}$ & 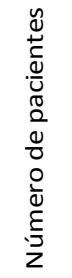 & 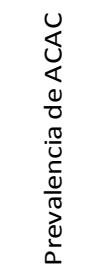 & 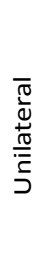 & $\begin{array}{l}\bar{\pi} \\
\frac{\pi}{0} \\
\frac{\pi}{\bar{\omega}}\end{array}$ & 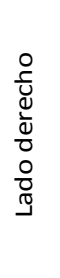 & $\begin{array}{l}\frac{0}{0} \\
\frac{0}{U} \\
\frac{O}{T} \\
\frac{0}{N} \\
\frac{0}{0} \\
\frac{0}{0}\end{array}$ & $\begin{array}{l}\overline{0} \\
\frac{\pi}{0} \\
\dot{w}\end{array}$ & 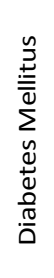 & 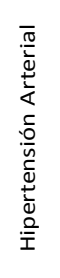 & 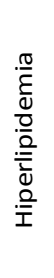 & \begin{tabular}{l}
$\frac{1}{0}$ \\
$\frac{0}{0}$ \\
$\frac{\pi}{5}$ \\
\multirow{1}{3}{}
\end{tabular} & $\begin{array}{l}0 \\
\frac{\pi}{0} \\
\frac{0}{y} \\
0 \\
0 \\
0\end{array}$ \\
\hline Lee et al 2014 & FyM & $64.9^{*}$ & 4050 & $6 \% * *$ & NR & NR & NR & NR & si & si & no & si & no & no \\
\hline Imanmoghaddam et al 2012 & FyM & $55.47^{*}$ & 960 & $1.97 \% * *$ & 5 & 10 & 7 & 9 & si & no & no & no & no & no \\
\hline Sens et al 2012 & FyM & Más de 40 & 21 & $80.95 \%$ & NA & NA & NA & NR & NR & NR & NR & NR & NR & NR \\
\hline Baryam et al 2006 & FyM & Más de 40 & 4106 & $2.14 \%^{* *}$ & 70 & 18 & 20 & 50 & si & no & si & no & no & no \\
\hline Baumann et al 2012 & FyM & Más de 50 & 4007 & $3.59 \%$ & NR & NR & 19 & 18 & si & no & no & no & no & no \\
\hline Khambete et al 2014 & FyM & $64.45^{*}$ & 50 & $34 \%$ & NR & NR & NR & NR & NR & NR & NR & NR & NR & NR \\
\hline Sisman et al 2007 & FyM & Más de 40 & 750 & $5.06 \% * *$ & 26 & 12 & NR & NR & no & si & si & si & si & no \\
\hline Yoon et al 2008 & FyM & 65.2 & 110 & $15 \%$ & NR & NR & NR & NR & NR & NR & NR & NR & NR & NR \\
\hline Ezoddini et al 2014 & FyM & $45.13^{*}$ & 1682 & $2.43 \% * *$ & 27 & 14 & NR & NR & no & no & si & no & no & no \\
\hline
\end{tabular}

(si) relacionado, (no) no relacionado, Femenino (F), Masculino (M), ${ }^{*}$ edad promedio, ${ }^{* *}$ más prevalente en mujeres, ${ }^{* * *}$ más prevalente en hombres

Tabla 2. Prevalencia de ACAC en Panorámicas en pacientes con enfermedades sistémicas

\begin{tabular}{|c|c|c|c|c|c|c|c|}
\hline Autores & Sexo & Edad & $\begin{array}{l}\text { Número de } \\
\text { pacientes }\end{array}$ & Enfermedad sistémica & $\begin{array}{l}\text { Prevalencia } \\
\text { de ACAC }\end{array}$ & Factores de riesgo & Referencia \\
\hline Hamedani et al & $\mathrm{F}$ & $54.1^{*}$ & 78 & Postmenopausia & $15 \%$ & NR & 22 \\
\hline Friedlander et al & $\mathrm{F}$ & Mayores de 50 & 481 & Postmenopausia & $7 \%$ & No hallaron asociación & 23 \\
\hline Freidlander et al & $\mathrm{F}$ & Mayores de 50 & 571 & Postmenopausia & $6.30 \%$ & Edad & 24 \\
\hline Uthman et al & $\mathrm{F}$ & NR & 45 & Posmenopausia & $1.50 \%$ & HA y Diabetes & 25 \\
\hline David et al & $\mathrm{F}$ & De 48 a 76 & 44 & Postmenopausia & $43.20 \%$ & HA e hiperlipidemia & 26 \\
\hline Friedlander et al & $\mathrm{F}$ & Mayores de 50 & 44 & Postmenopausia & $7.00 \%$ & Densidad de masa ósea & 27 \\
\hline Guerreiro et al & Fy $M$ & $40.73^{\star}$ & 300 & SIDA & $8.33 \%$ & Tipo de antiretrovirales & 28 \\
\hline Freidlander et al & F y $M$ & $73.2^{*}$ & 60 & Hiperparatoroidismo & $40 \%$ & HA y dislipidemia & 29 \\
\hline MacDonald et Al & $M$ & 64 & 1 & Hiperparatoroidismo & NR & Enfermedad renal & 30 \\
\hline Gokce et al & F y $M$ & $45.5^{*}$ & 95 & Enfermedad renal terminal & $27 \%$ & Dialisis peritoneal & 31 \\
\hline Amahd et al & F y $M$ & $57^{\star}$ & 75 & Enfermedad renal & $29 \%$ & Hemodiálisis & 32 \\
\hline Gunnarsson et al & F y $M$ & $47.6^{*}$ & 790 & Apnea del sueño & NR & No hallaron asociación & 33 \\
\hline Chang et al & F y $M$ & $58.5^{*}$ & 148 & Síndrome Z & $38.50 \%$ & C, triglicéridos, colesterol, gluco & 34 \\
\hline Pornprasertuk et al & Fy M & NR & 85 & Síndrome metabólico & NR & $\begin{array}{c}\text { Obesidad, hipertensión, } \\
\text { dislipidemia, resistencia a la } \\
\text { insulina. }\end{array}$ & 35 \\
\hline Tofangchiha et al & F y $M$ & De 35 A 72 & 458 & Diabetes tipo II & $11.70 \%$ & Factores metabólicos & 36 \\
\hline Khosropanah et al & F y $M$ & $55.5^{\star}$ & 84 & Enfermedad coronaria & $32.10 \%$ & $\mathrm{NR}$ & 37 \\
\hline Freidlander et al & M & 74 & 1 & $\begin{array}{c}\text { Carcinoma de células } \\
\text { escamosas }\end{array}$ & NR & Radioterapia & 38 \\
\hline
\end{tabular}

Femenino $(\mathrm{F})$, Masculino $(\mathrm{M}),{ }^{*}$ edad promedio, $\mathrm{IMC}=$ índice de masa corporal, HA=Hipertensión arterial, NR=No reporta 
Tabla 3. Prevalencia de ACAC en Panorámicas en pacientes con enfermedad periodontal

\begin{tabular}{|c|c|c|c|c|c|}
\hline Autores & Sexo & Edad & $\begin{array}{l}\text { Número de } \\
\text { pacientes }\end{array}$ & Conclusión & $\begin{array}{l}\text { Número de } \\
\text { referencia }\end{array}$ \\
\hline Ramesh et al & FyM & 30 a 94 & 419 & $\begin{array}{l}\text { Existe asociación entre la presencia de ACAC y osteopenia, } \\
\text { más no con pérdida ósea }\end{array}$ & 39 \\
\hline David et al & $\mathrm{F}$ & 45 a más & 84 & En presencia de $A C A C$ hay mayor pérdida de altura ósea & 40 \\
\hline Chin et Al & FyM & 40 a 69 & 749 & $\begin{array}{l}\text { Los pacientes con periodontitis crónica padecen } \\
\text { ateroesclerosis subblínica. }\end{array}$ & 45 \\
\hline Cairo et al & FyM & Menos de 40 & 180 & $\begin{array}{l}\text { La profundidad del sondaje y el sangrado son predictores } \\
\text { de patologías cardiovasculares }\end{array}$ & 46 \\
\hline Cairo et al & FyM & 18 a 40 & 90 & $\begin{array}{l}\text { Periodontitis está asociada a ateroesclerosis subclínica en } \\
\text { pacientes sanos menores de } 40 \text { años }\end{array}$ & 47 \\
\hline Shin et al & FyM & Mayores de 50 & 5404 & $\begin{array}{l}\text { La pérdida de dientes por enfermedad oral es predictora } \\
\text { ateroesclerosis subclínica }\end{array}$ & 41 \\
\hline Puhar et al & FyM & $57^{*}$ & 128 & $\begin{array}{l}\text { Se demostró que los cambios en la Carótida están } \\
\text { asociados a periodontitis crónica }\end{array}$ & 44 \\
\hline Yu et al & FyM & $70^{*}$ & 847 & $\begin{array}{l}\text { Se halló asociación entre la hiperglicemia y la higiene oral } \\
\text { pobre con el aumento de grosor de la íntima y la presencia } \\
\text { de placas ateromatosas }\end{array}$ & 42 \\
\hline Morado et al & FyM & Mayores de 18 & 50 & $\begin{array}{l}\text { Mientras más severo sea el caso de periodontitis, más } \\
\text { relacionado estará a la presencia de ateroesclerosis. }\end{array}$ & 43 \\
\hline
\end{tabular}

Femenino (F), Masculino (M), ${ }^{*}=$ media

Tabla 4. Radiopacidades que pueden causar confusión en la detección de ACAC en radiografías Panorámicas

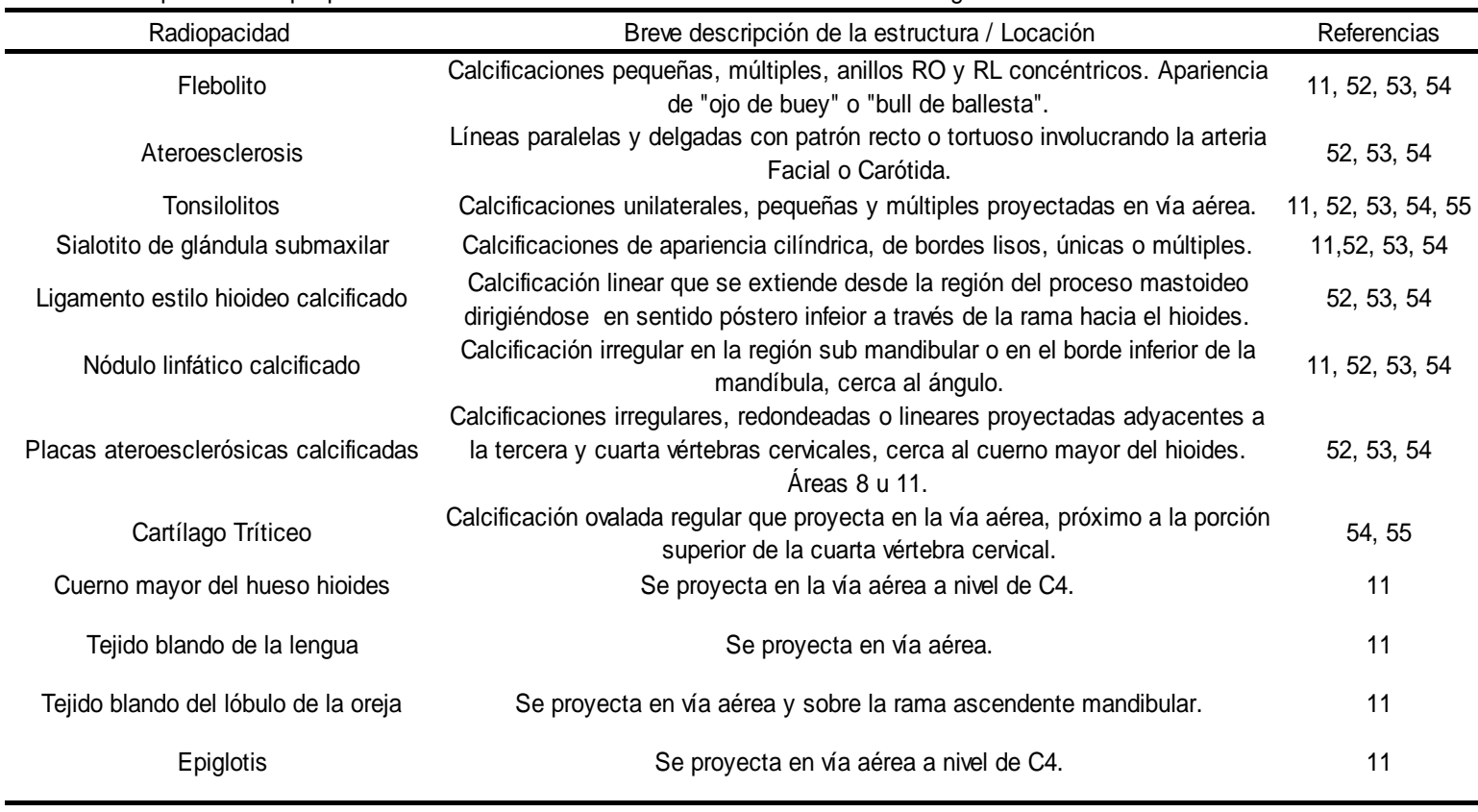




\section{REFERENCIAS BIBLIOGRÁFICAS:}

1. Castelo L, Aladro F, Licea M, Hernández J, Arnold Y. Factores de riesgo y diagnóstico de la enfermedad carotídea. Rev Peru Epidemiol. 2013; 17 (1): 1-7.

2. Sens J, Quiroz T, Barreto S, Felice K, Ferreira F, Figueredo A. Sensitivity and accuracy of panoramic radiography in identifying calcified carotid atheroma plaques. Braz J Oral Sci. 2012; 11 (2): 88-93.

3. Silveira Mariana, Cardozo R, Da Silva P, Alvares A, Rubira I. Contribução da radiografia panorámica no diagnóstico de calcificação de ateroma de carótida: relato de caso e revisão da literatura. Rev Port Estomatol Med Dent Cir Maxilofac. 2015; 56 (2): 127-31.

4. Cunnane E, Mulvihill J, Barrett $\mathrm{H}$, Healy D, Kavanagh E, Walsh S, Walsh M. Mechanical, biological and structural characterization of human atherosclerotic femoral plaque tissue, Acta Biomaterialia, 2015; 11 ( 1): 295-303.

5. Roldán R, Oñate R, López F, Cabrerizo M, Martínez F. Panoramic radiograph a method for detecting calcified ateroma plaques. Review of literature. Med Oral Patol Oral Cir Bucal. 2006; 11: E261-6.

6. Imanimoghaddam M, Rah M, Mahmaoudi E, Javadzade A. Doppler sonography confirmation in patients showing calcified carotid artery atheroma in panoramic radiography and evaluation of related risks factors. J Dent Res Dent Clin Dent Prospects. 2012; 6 (1): 6-11

7. INEl. Condiciones de vida en el Perú. №2. 2013

8. Vilar G, Villanueva M, Tenorio F, Díaz J, De La Fuente J, Sánchez B. Identification of calcified atheromatous plaques from patients attending a dental clinic unit in León, México. Dent Oral Craniofac Res. 2015; 1 (29): 53-5.

9. Alzoman H, Al-Sadham R, Al-Labem Z, Al-Sakaker A, Al-Fawaz Y. Prevalence of carotid calcification detected on panoramic radiographs in a Saudi population from a training institute in Central Saudi Arabia. Saudi Med J. 2012; 33 (2): 177-81.

10. Atalay Y, Asutay F, Serkan K, Koparal M, Adali F, Gulsun B. Evaluation of calcified carotid atheroma on panoramic radiographs and Doppler ultrasonography in an population. Clin Interv Anging. 2015; 8 (10): 1121-9.

11. Manzi F, Bóscolo F, De Almeida S, Haiter F. Panoramic radiography as an auxiliary in detecting patients at risck of cerebrovascular accident (CVA): a case report. J Oral Sci. 2003; 45 (3): 177-80.

12. Guimarães J, Kreich E, Baldani M, Luciano M, De Melo J, De Moraes L. Panoramic radiography in the diagnosis of carotid artery atheromas and the associated risk factors. Open Dent J. 2011; 5: 79-83.

13. Lee J, Kim O, Chung H, Kim Y, Kweon S, Lee $Y$ et al. The correlation of carotid artery calcificaction on panoramic radiographs and determination of carotid artery atherosclerosis with ultrasonography. Oral Surg Oral Med Oral Patol Oral Radiol. 2014; 118: 739-45.

14. Sens J, Queiroz T, Barreto S, Felice K, Ferreira F, Figuereido A. Sensitivity and accuracyof panoramic radiography in identifiying calcified carotid atheroma plaques. Braz J Oral Sci. 2012; 11(2): 88-93.

15. Baryam B, Uckan S, Acikgoz A, Müderrisoglu H, Aydinalp A. Digital panoramic radiography: a realiable method to diagnose carotid artery atheromas. Dentomaxillofac Radiology. 2006; 35: 266-70.

16. Alves N, Deana N, Garay I. Review article. Detection of common carotid artery calcifications on panoramic radiographs: prevalence and reliability. Int Clin Exp Med. 2014; 7 (8): 1931-9.

17. Baumann S, Meier R, Burow A, Lyrer P, Engelter S, Bonati L et al. Reconizing calcifications of the Carotid artery oh panoramic radiographs to prevent strokes. Research and Science. 2012; 122: 1-8.

18. Khambete N, Kumar R, Risbud M, Joshi A. Reliability of digital panoramic radiographs in detecting calcified carotid artery atheromatous plaques: A clinical study. Indian J Dent Res. 2014; 25 (1): 36-40.

19. Sisman Y, Tarim E, Gokce C, Menku A, Ulker M, Akgunlu F. The prevalence of carotid artery calcification on the panoramic radiographs in Cappadocia región population.Eur J Dent. 2007; 1: 132-8. 
20. Yoon S, Yoon W, Kim O, Lee J, Knag B. Diagnostic accuracy of panoramic radiography in the detection of calcified carotid artery. 2008; 37: 104-8.

21. Ezoddini F, Mirzaei M, Nayer S, Beshrati S, Moeini M. Evaluation of positive predictive value for digital panoramic radiography in comparison to ultrasound in the diagnosis of calcified carotid atheroma. Health. 2014; 6: 1933-40.

22. Hamedani S, Dabbaghmanesh M, Zare Z, Hasani M, Ardakani M, Hasani M et al. Relationship of elongated styloid process in digital panoramic radiography with carotid intima thickness and carotid atheroma in Doppler ultrasonography in osteoporotic females. J nDent Shiraz Univ Med Sci. 2015; 16 (2): 93-9.

23. Friedlander A, El- Saden S, Hazboun, Chang T, Wong W, Garrett N. Detection of carotid artery calcification on the panoramic images of post-menopausal females is significantly associated with severe abdominal aortic calcification: a risk indicator of future adverse vascular events. Dentomaxillofac Radiol. 2015; 44 (7): 20150094

24. Friedlander A, El-Saden S, Aghazadehsanai N, Chang, T, Harada N, Garret. Association of calcified carotid ateromas visualized on panoramic images and aortic calcifications seen on chest radiographs of postmenopausal women. JADA. 2014; 145 (4): 345-51.

25. Uthman A, Al-Naqeeb L, Al-Saleem, Al Rawi N. Carotid artery atheromas and calcifications among postmenopausal women with diabetes and hypertension. J Int Dent Med Res. 2014; 7 (2): 42-8.

26. David M, Usha V. Prevalence of carotid artery ateromas in post-menopausal women: a digital panoramic radiographic study. J Indian Acad Oral Med Radiol. 2013; 25 (0): 1-6.

27. Friedlander A, Chang T, Aghazadehsanai N, Berenji G, Harada N, Garrett. Panoramic images of white and black post-menopausal females evidencing carotid calcifications are at high risck of comorbid osteopenia of the femoral neck. Dentomaxillofac Radiol. 2013; 42: 20120195.

28. Guerreiro N, Nelo E, Mesquita F, Wamling L, Ortega K. Prevalence of calcified carotid artery ateromas in panoramic radiographs of HIV-positive patients undergoing antiretroviral treatment: a retrospective study. Oral Surg Iral Med Oral Pathol Oral Radiol. 2014; 117:67-74.

29. Friedlander A, Aghazadehsanai N, Chang T, Harada N, Garret N. Prevalence of calcified carotid artery ateromas on panoramic imagens of individuals with primary hyperparathyroidism. Dentomaxillofac Radiol. 2013; 42: 20130118.

30. MacDonal d, Zhang L, Gu Y. Case report: Calcification of the external carotid arteries and their branches. Dentomaxillofac Radiol. 2012; 41: 615-8.

31. Gokce C, Sisman Y, Sipahioglu M, Ertas E, Akgunlu F, Unal A et al. The prevalence of carotid artery calcification on the panoramic radiographs of end-satge renal disease patients with peritoneal dialysis: Do incidental findings provide life-saving information? J Int Med Res. 2008; 36: 47-53.

32. Ahmad A, Sadat M, Asadsadeh R, Mohammad A, Alatab S, Miri R, Yaseri M. Differences in cardiovascular disease risk factors associated with maximum and mean carotid intima-media thickness amog hemodialysis patients. Iran J Kidney Dis. 2012; 6: 203-8.

33. Gunnarsson S, Peppard P, Korcarz C, Barnet J, Aeschlimann S, Hagen E et al. Obstructive sleep apnea in associated with future subclinical carotid artery disease. Arterioscler Thromb Vasc Biol. 2014; 34: 233842.

34. Chang T, Tanner J, Harada N, Garret N, Friedlander A. Prevalence of calcified carotid artery atheromas on the panoramic images of patients with syndrome Z, coexisting obstructive sleep apnea, and metabolic syndrome. Oral Surg Oral Med Oral Pathol Radiol. 2012; 113: 134-41.

35. Pornprasertsuk S, Virayavanich W, Thanakum S, Siriwongpairat P, Amaekchok P, Khovidhunkit W. Carotid atheroma detected by panoramic radiography and ultrasonography in patients with metabolic syndrome. Oral Radiol. 2011; 27: 43-9.

36. Tofangchiha M, Foroozia M, Bakhshi M, Bashizade $\mathrm{H}$. The carotid artery calcification in tipe II diabetic patients on panoramic radiographs: An important marker for vascular risk. Sci Res Essays. 2011; 6 (31): 6548-53.

37. Khosropanah S, Shahidi S, Bronoosh P, Rasekhi A. Evaluation of catorid calcification detected using panoramic radiography and carotid Doppler sonography in patients with and without coronary artery disease. Br Dent J. 2009; 207 (4): 1-4. 
38. Friedlander A, Federico M, Yueh R, Norman K, Chin E. Radiation-associated carotid artery atheroesclerosis: case report and review of contemporaneous literature. Spec Care Dentist. $2009 ; 29$ (2): 75-9.

39. Ramesh A, Soroushian S, Ganguly R. Coincidence of calcified carotid atheromatous plaque, osteoporosis, and periodontal bone loss in dental panoramic radiographs. Imaging Sci Dent. 2013; 43: 235-43.

40. David M, Saxena R, Indira A, Mahalakshme I. Correlation between carotid artery calcifications and periodontitis in posmenopausal women: a retrospective digital panoramic radiographic study. AOSR. 2012; $2(2): 71-6$.

41. Shin J, Kweon K, Lee $Y$, Kim Y, Ching $H$. Relationship between periodontal disease and subclinical atherosclerosis: The Dong-gu study. J Clin Periodontol. 2014; 41: 262-8.

42. Yu H, Qi L, Liu L, Wang X, Zhang Y, Huo $Y$ et al. Association of Carotid intima.media thickness and atherosclerotic plaque with periodontal status. J Dent Res. 2014; 93 (8): 744-51.

43. Morado M, Faria R, Azevedo E, Conceição M, Martins L. Periodontitis and aterosclerosis: an observational study. J Periodont Res. 2013; 48: 452-57.

44. Puhar I, Lovrencic A, Sodec D, Strineka M, Bozic D, Plancak D. Carotid Intima-Media Thicness in patients with chronic and aggressive periodontitis. Acta Stomatol Croat. 2012; 46 (4): 255-62.

45. Chin U, Ji S, Lee S, Ryu J, Lee J, Shin C et al. Relationship between tooth loss and carotid intima-media thickness in Korean adults. J Adv Prosthodont. 2010; 2: 122-7.

46. Cairo F, Nieri M, Gori A, Castellani S, Abbate R, Pini G. Periodontal variables may predict sub clinical atherosclerosis and systemic inflammation in young adults. Eur J Orla Implantol. 2009; 2 (2): 125-33.

47. Cairo F, Castellani S, Gori A, Nieri M, Baldelli G, Abbate R. Severe periodontitis in young adults is associated with subclinical atherosclerosis. J Clin Periodontol. 2008; 35: 465-72.

48. Vaish S, Menon I. Review article: Periodontitis and cardiovascular diseases-a review on causality hypotheses. Int J Public Health. 2011; 2 (1): 13-8.

49. Tonetti M. Periodontitis and risck for atherosclerosis: an update on intervention trials. J Clin Periodontol. 2009; 36 (10): 15-9.

50. Alves N, Deana N, Garay I. Review article: Detection of common carotid artery calcifications on panoramic radiographs: prevalence and reliability. Int Clin Exp Med.2014; 7 (8): 1931-9.

51. Rubin A. Radiografia panorâmica e calcificações em tecidos moles. Monografía para la obtención del título de Especialista en Radiología Odontológica e Imaginología. 2011. Universidad Federal do Rioz Grande do Sul. Facultad ed Odontología. Especialização em Radiologia Odontológica e Imaginologia.

52. Vengalath J, Homberhalli J, Rajkumar B, Channaiah G. Prevalence of soft tissue calcifications on digital panoramic radiografphs: A retrospective study. J Indian Acade Oral Med Radiol. 2014; 26 (4): 385-89.

53. Bayer S, Helrgen E, Bös C, Kraus D, Enkling N, Mues S. Prevalence of findings compatible with carotid artery calcifications on dental panoramic radiographs. Clin Oral Invest. 2011; 15: 563-9.

54. Saga R, Fenyo M, Fernandes A, Meurer M. Study of the localization of radiopacities similar to calcified carotid ateroma by means of panoramic radiography. Oral Surg Oral Med Oral Pathol Oral Radiol Endod. 2006; 101: 374-8.

55. Ahmad M, Madden R, Pérez O. Triticeous cartilage: Prevalence on panoramic radiographs and diagnostic criteria. Oral Surg Oral Med Oral Pathol Oral Radiol Endod. 2005; 99: 225-30.

56. Garay I, Olate S. Consideraciones actuales en el estudio imagenológico de las calcificaciones de tejidos blandos en la zona de ángulo mandibular. Int J Odontostomat. 2013; 7 (3): 455-64.

57. Alves C, Pimenta T, Neves E, Alves R. Bilateral tonsilloliths and calcified carotid atheromas: case report and literature review. J Craniomaxillofac Surg.2013;41:179-82. 